\title{
Estimativa da evapotranspiração de referência do Estado de Sergipe
}

\author{
Reference evapotranspiration estimation of the State of Sergipe
}

\author{
R. R. Gomes Filho ${ }^{1 *}$; L. G. de Souza1; I. L. N. Santos ${ }^{1}$; K. V. Santos ${ }^{1}$; D. T. B. de \\ Oliveira $^{1}$; D. T. da Silva ${ }^{1}$ \\ ${ }^{1}$ Departamento de Engenharia Agrícola, Universidade Federal de Sergipe, CEP 49100-000, São Cristovão-Sergipe, \\ Brasil
}

*rrgomesfilho@hotmail.com

(Recebido em 19 de junho de 2017; aceito em 30 de setembro de 2017)

\begin{abstract}
Aperfeiçoar a gestão dos recursos hídricos é o primeiro passo para sua conservação. Nesse sentido, a evapotranspiração de referência, como uma das componentes do balanço de água, é de grande utilidade em climatologia, para a quantificação das disponibilidades hídricas regionais [1]. A estimativa das perdas por evapotranspiração é de grande importância para atividades como projetos de irrigação, gerenciamento de reservatórios e planejamento de uso e outorga de recursos hídricos, entre outras [2]. Apesar da existência de diversos métodos para estimativa da evapotranspiração de referência (ETo), eles, no entanto, são utilizados em condições climáticas e agronômicas muito diferentes, em função da disponibilidade de dados climáticos. O método de Penman Monteith é considerado como padrão, porém para sua utilização há necessidade de algumas variáveis meteorológicas que podem não estar disponíveis no local. Portanto, o objetivo deste trabalho foi correlacionar os métodos de estimativas de ETo obtidos pelo método padrão com os métodos de Linacre, Blaney-Criddle, Hargreaves-Samani e Camargo, que utilizam menor número de variáveis climatológicas. A estimativa da ETo foi determinada no período de maio de 2014 a abril de 2017 para os municípios de Carira, Itabaianinha e Poço Verde, que dispõem de estações meteorológicas automáticas do Instituto Nacional de Meteorologia (INMET) e ocupam as três mesorregiões de Sergipe. Verificou-se que o método de Hargreaves-Samani foi o que obteve melhor desempenho e uma correlação muito alta para os municípios de Carira e Itabaianinha. O método de Blaney-Criddle apresentou a maior correlação, porém não apresentou resultado satisfatório em relação ao desempenho para os municípios estudados.
\end{abstract}

Palavras-chave: Penman-Monteith, equações empíricas, dados climáticos.

Improving the management of water resources is the first step towards its conservation. In this sense, reference evapotranspiration, as one of the components of the water balance, is of great use in climatology, for the quantification of regional water availability [1]. The estimation of losses through evapotranspiration is of great importance for activities such as irrigation projects, reservoir management and planning of water use and granting, among others [2]. Despite the existence of several methods to estimate reference evapotranspiration (ETo), they are, however, used in very different climatic and agronomic conditions, depending on the availability of climatic data. The Penman Monteith method is considered to be standard, but for its use there is a need for some meteorological variables that may not be available locally. Therefore, the objective of this work was to compare the values of ETo estimates obtained by the standard method with the methods of Linacre, Blaney-Criddle, Hargreaves-Samani and Camargo, which use fewer climatological variables. The ETo estimate was determined from May 2014 to April 2017 for the cities of Carira, Itabaianinha and Poço Verde, which have automatic meteorological stations of the National Institute of Meteorology (INMET) and occupy the three mesoregions of Sergipe. It was verified that the HargreavesSamani method was the best performance and a very high correlation for Carira and Itabaianinha municipalities. The Blaney-Criddle method presented the higher correlation, but it didn't present satisfactory results regarding the performance for the municipalities of Carira, Itabaianinha and Poço Verde.

Keywords: Penman-Monteith, empirical equations, climatic data. 


\section{INTRODUÇÃO}

Sergipe é o menor estado brasileiro em extensão territorial dividido em três mesorregiões, Agreste, Leste e Sertão. A principal atividade agrícola de Sergipe é o cultivo de cana-de-açúcar, da qual são produzidas mais de 1.400 .000 toneladas por ano. Além da cana, são cultivados mandioca, laranja, coco, feijão, milho e arroz, sendo que grande parte é cultivada sem irrigação, porém a ocorrência de longas estiagens em grande parte do estado sergipano é responsável pela redução do nível de água nos reservatórios aumento o risco da agricultura de sequeiro.

A irrigação tem sido uma alternativa para garantir a produção agrícola, porém em virtude da baixa disponibilidade hídrica da região, é necessário o uso racional da água, ou seja, realização de um manejo adequado da irrigação.

Uma informação muito importante para auxiliar o manejo da água na irrigação é o conhecimento do consumo hídrico das culturas baseado na estimativa da evapotranspiração de referência, visto que atualmente existe uma maior conscientização da população no uso adequado dos recursos hídricos, quando se compara a períodos anteriores [3].

A evapotranspiração de referência (ETo) é uma variável muito importante para o dimensionamento e manejo de sistemas de irrigação, pode ser obtida facilmente em função de ser afetada apenas por fatores climáticos. De uma maneira geral, os métodos de estimativa da evapotranspiração de referência se baseiam em variáveis climáticas.

A ETo pode ser calculada através de métodos diretos e indiretos. O método direto é aquele que utiliza lisímetro, mesmo apresentando ótimos resultados, o custo dos equipamentos é alto, tornando impraticável o seu uso no manejo de irrigação diário [4]. Os métodos indiretos são calculados utilizando o tanque classe A ou através de fórmulas preestabelecidas, como as de Camargo, Linacre, Hargreaves-Samani, Blaney-Criddle entre outras. O método de PenmanMonteith, que leva em consideração aspectos termodinâmico e aerodinâmico da cultura, é considerado como modelo padrão para quantificar a demanda atmosférica de evapotranspiração [2]. Na utilização do método de Penman-Monteith para estimar a ETo existe uma quantidade de variáveis atmosféricas superiores aos dos outros métodos, que muitas vezes não estão disponíveis em condições reais e vários estudos têm sido realizados com a finalidade de estabelecer uma relação dos valores estimados por outros métodos com o método de Penman-Monteith e dados experimentais nas regiões específicas $[2,5]$.

A grande dificuldade dos produtores é a obtenção dos dados que geralmente não estão disponíveis e uma série histórica incompleta, sendo que muitas vezes, não é possível utilizar o método padrão de Penman-Monteith. No estado de Sergipe tem cinco estações meteorológicas automáticas ligadas ao INMET - Instituto Nacional de Meteorologia, localizadas em cinco municípios do estado, distribuídos nas três mesorregiões, onde é possível a obtenção dos dados.

Baseado no exposto acima, este trabalho teve como objetivo estimar a ETo diária, realizar comparações dos métodos de estimativa da ETo e a análise da variabilidade espacial e temporal no estado de Sergipe, levando em consideração os dados meteorológicos dos municípios de Carira, Itabaianinha e Poço Verde.

\section{MATERIAL E MÉTODOS}

Os dados diários de temperatura, umidade, pressão, velocidade do vento, radiação solar e insolação foram obtidos em estações meteorológicas automáticas do Instituto Nacional de Meteorologia - INMET localizadas nos municípios de Carira, Itabaianinha e Poço Verde no estado de Sergipe. Estes estão localizados em mesorregiões diferentes.

O município de Carira localiza-se na mesorregião do Sertão, na região oeste do estado de Sergipe. É o segundo maior produtor de milho do estado de Sergipe [6].

O município de Itabaianinha localiza-se na mesorregião do Leste. É o maior produtor de citros do estado de Sergipe. [6]. Já A região de Poço Verde, localizada na mesorregião do Agreste, é a maior produtora de feijão do Estado de Sergipe e a décima segunda do Brasil, sendo a segunda maior produtora de milho irrigado no Estado [6].

Foi utilizado o software EToCalc para estimativa da ETo pelos métodos de Camargo, Linacre, Hargreaves-Samani, Blaney Criddle e Penman-Monteith. Os dados climatológicos do período de 
maio de 2014 a abril de 2017 foram tabulados e estimados os valores de Evapotranspiração de Referência (ETo) pelos métodos empíricos citados a seguir.

O método de Camargo (1971) [7] foi uma simplificação do método de Thorntwaite (1948) [8]. Apresenta como vantagem a utilização apenas da temperatura média do ar e como desvantagem não considerar o poder evaporante do ar que o método original de Thorntwaite se baseia. A vantagem adicional deste método é que não há necessidade do uso de dados normais de temperatura do ar. A ETo é estimada diretamente pela seguinte equação:

$\mathrm{ET}_{\mathrm{o}}=0,01 \mathrm{Q}_{\mathrm{o}} \mathrm{T}_{\text {med }}$

em que: $Q_{o}$ é a radiação solar extraterrestre (tabelado em função da latitude do local e do mês), expressa em mm de evaporação equivalente, $\mathrm{T}_{\mathrm{med}}$ é temperatura média do período considerado, em ${ }^{\circ} \mathrm{C}$.

O método de Hargreaves-Samani foi desenvolvido para as condições climáticas de clima semiárido da Califórnia em 1985. É recomendado pela FAO [9] como uma opção para a estimativa de ETo, quando há somente disponibilidade de dados de temperatura do ar. Não é de aplicação universal, portanto, deve ser calibrado para outras condições climáticas. Normalmente, provoca superestimativas em condições de clima úmido [9]. Hargreaves e Samani aplicando a análise de regressão em dados diários de evapotranspiração de referência de Davis-Califórnia obtiveram a seguinte equação:

$\mathrm{ET}_{\mathrm{o}}=0,0023\left(\mathrm{~T}_{\text {med }}+17,8\right)\left(\mathrm{T}_{\max }-\mathrm{T}_{\min }\right)^{0,5} \mathrm{R}_{\mathrm{a}}$

em que: $\mathrm{T}_{\mathrm{med}}, \mathrm{T}_{\max }$ e $\mathrm{T}_{\min }, \mathrm{em}{ }^{\circ} \mathrm{C}$, representam, respectivamente, temperaturas média, máxima e

mínima e $\mathrm{R}_{\mathrm{a}}$ é a radiação solar no topo da atmosfera $\left(\mathrm{mm} \mathrm{dia}^{-1}\right)$, obtida por equação ou tabelas.

O método de Blaney Criddle foi desenvolvido para uma região semiárida do Novo México e Texas localizado no oeste dos Estados Unidos da América em 1950. Doorenbos \& Pruitt (1984) [10] propuseram a aplicação de um fator de correção, utilizando a umidade, velocidade do vento e insolação para aplicação do método em várias condições climáticas [11]. A ETo é estimada diretamente pela seguinte equação:

$\mathrm{ET}_{\mathrm{O}}=\mathrm{c}[(0,457 \mathrm{~T}+8,13) \mathrm{P}]$

em que: c é o coeficiente regional de ajuste da equação (tabelado em função do brilho solar, velocidade do vento e umidade relativa mínima); T é a temperatura média mensal, em ${ }^{\circ} \mathrm{C}$ e $\mathrm{P}$ é a percentagem mensal de horas anuais de luz solar.

O método de Linacre foi originalmente testado para as condições climáticas da Austrália, é um método baseado na simplificação do método de Penman-Monteith, utilizando dados de temperaturas (máxima, mínima e ponto do orvalho) em função da latitude e altitude [11]. A equação de estimativa de ETo pelo método de Linacre (1977) [12] é:

$\mathrm{ET}_{\mathrm{O}}=\frac{700 \frac{\left(\mathrm{T}_{\mathrm{med}^{+}}{ }^{0,006 \mathrm{z})}\right.}{(100-\varnothing)}+15\left(\mathrm{~T}_{\text {med }}-\mathrm{T}_{\mathrm{d}}\right)}{\left(80-\mathrm{T}_{\mathrm{med}}\right)}$

O método de Penman-Monteith considera que a evapotranspiração de referência incorpora os aspectos aerodinâmico e termodinâmico, inclui na sua dedução a resistência ao fluxo de calor sensível e vapor da água [13]. É recomendado pelo FAO como método padrão [9] para estimativa da ETo, descrita pela equação:

$\mathrm{ET}_{\mathrm{o}}=\frac{0,408 \Delta\left(\mathrm{R}_{\mathrm{n}}-\mathrm{G}\right)+\gamma \frac{900}{\mathrm{~T}+273} \mathrm{U}_{2}\left(\mathrm{e}_{\mathrm{s}}-\mathrm{e}_{\mathrm{a}}\right)}{\Delta+\gamma\left(1+0,34 \mathrm{U}_{2}\right)}$

em que: $\Delta$ é a declividade da curva de pressão de vapor de saturação, em $\mathrm{kPa}^{\circ} \mathrm{C}^{-1} ; \mathrm{R}_{\mathrm{n}}$ é o saldo de radiação à superfície, em $\mathrm{MJ} \mathrm{m}^{-2} \operatorname{dia}^{-1} ; \mathrm{G}$ é o fluxo de calor no solo, em $\mathrm{MJ} \mathrm{m}^{-2} \operatorname{dia}^{-1} ; \gamma$ é a constante psicrométrica, em $\mathrm{kPa}^{\circ} \mathrm{C}^{-1}$; $\mathrm{T}$ é a temperatura do ar a $2 \mathrm{~m}$ de altura, em ${ }^{\circ} \mathrm{C} ; \mathrm{U}_{2}$ é a velocidade do vento à altura de $2 \mathrm{~m}$, em m s${ }^{-1}$; e é a pressão de saturação de vapor, em $\mathrm{kPa}$; $\mathrm{e}_{\mathrm{a}}$ é a pressão de vapor atual do ar, em $\mathrm{kPa}$.

Para comparação e análise dos resultados foram utilizados critérios envolvendo o erro padrão de estimativa (EPE), erro padrão da estimativa ajustado (EPEa), coeficientes de ajustes das equações lineares com seus respectivos coeficientes de determinação $\left(\mathrm{R}^{2}\right)$, [14].

$\mathrm{EPE}=\left(\frac{\sum\left(\mathrm{Y}_{\mathrm{i}}-\mathrm{Y}_{\mathrm{m}}\right)^{2}}{\mathrm{n}-1}\right)^{0,5}$ 
$\mathrm{EPE}_{\mathrm{a}}=\left(\frac{\sum\left(\mathrm{Y}_{\mathrm{ic}}-\mathrm{Y}_{\mathrm{m}}\right)^{2}}{\mathrm{n}-1}\right)^{0,5}$

em que: $Y_{i}$ é a evapotranspiração estimada pelos métodos de Linacre, Hargreaves-Samani, Blaney-Criddle e Camargo $\left(\mathrm{mm} \mathrm{dia}^{-1}\right), \mathrm{Y}_{\mathrm{m}}$ é a evapotranspiração estimada pelo método de Penman-Monteith $\left(\mathrm{mm} \mathrm{dia}^{-1}\right)$, n é o número total de observações e $\mathrm{Y}_{\mathrm{ic}}$ é a estimativa de ETo pelo método de Penman-Monteith, corrigida pelos coeficientes da regressão linear $\left(\mathrm{mm} \mathrm{dia}^{-1}\right)$.

As correlações entre os métodos de estimativas de evapotranspiração de referência foram realizadas estatisticamente através da equação linear:

$\mathrm{y}=\mathrm{ax}+\mathrm{b}$

em que: y representa a variável dependente; $\mathrm{x}$ a variável independente; $\mathrm{a}$ e $\mathrm{b}$ são coeficientes angular e linear da equação de regressão linear. A variável depende y corresponde aos valores estimados pelos métodos de Camargo, Hargreaves-Samani, Blaney-Criddle e Linacre, em quanto que a variável independente $\mathrm{x}$ corresponde aos valores obtidos pelo método padrão de PenmanMonteith.

A correlação entre os métodos de Camargo, Hargreaves-Samani, Blaney-Criddle, Linacre e o método padrão de Penman-Monteith foi realizada com base em indicadores estatísticos, a fim de se observar a precisão dada pelo coeficiente de correlação (r) que está associado ao desvio entre valores estimados e medidos indicando o grau de dispersão dos dados obtidos em relação à média. O coeficiente de correlação pode ser calculado a partir da seguinte equação:

$\mathrm{r}=\sqrt{\frac{\sum\left(\mathrm{Y}_{\text {est }}-\mathrm{Y}_{\text {med }}\right)^{2}}{\sum\left(\mathrm{Y}-\mathrm{Y}_{\text {med }}\right)^{2}}}$

em que: $Y_{\text {est }}$ representa a evapotranspiração estimada; $Y$ representa a evapotranspiração observada e $\mathrm{Y}_{\text {med }}$ representa a média das evapotranspirações observadas.

Os valores de coeficiente de correlação encontrados foram classificados de acordo com a metodologia de Hopkins (Tabela 1).

Tabela 1. Classificação das correlações de acordo com coeficiente de correlação (r)

\begin{tabular}{cc}
\hline Coeficiente de correlação $(\mathrm{r})$ & Classificação \\
\hline $0,0-0,1$ & Muito Baixa \\
$0,1-0,3$ & Baixa \\
$0,3-0,5$ & Moderada \\
$0,5-0,7$ & Alta \\
$0,7-0,9$ & Muito alta \\
$0,9-1,0$ & Quase perfeita \\
\hline
\end{tabular}

Aplicou-se, também, o teste desenvolvido por Willmott (1985) [15], que através de um coeficiente designado como concordância ou exatidão (d), quantifica matematicamente a dispersão dos dados em relação ao método padrão, sendo calculado pela equação:

$\mathrm{d}=1-\left[\frac{\sum\left(\mathrm{P}_{\mathrm{i}}-\mathrm{O}_{\mathrm{i}}\right)^{2}}{\sum\left(\mathrm{P}_{\mathrm{i}}-\mathrm{O}|+| \mathrm{O}_{\mathrm{i}}-\mathrm{O} \mid\right)^{2}}\right]$

em que: $\mathrm{P}_{\mathrm{i}}$ é a evapotranspiração estimada pelo método testado $(\mathrm{mm})$; $\mathrm{O}_{\mathrm{i}}$ é a ETo estimada pelo método padrão $(\mathrm{mm})$ e $\mathrm{O}$ é a média dos valores obtidos pelo método padrão (mm).

Com a correlação entre os dados e a exatidão, calculou-se o coeficiente de desempenho (c) (Tabela 2) que é determinado pelo produto do coeficiente de correlação e o de exatidão ( $\mathrm{c}=\mathrm{r} x$ d) $[16]$. 
Tabela 2. Valores do coeficiente de desempenho conforme Camargo e Sentelhas (1997) [16]

\begin{tabular}{cc}
\hline Valor de "c" & Desempenho \\
\hline$>0,85$ & Ótimo \\
$0,76-0,85$ & Muito Bom \\
$0,66-0,75$ & Bom \\
$0,61-0,65$ & Mediano \\
$0,51-0,60$ & Sofrível \\
$0,41-0,50$ & Mau \\
$\leq 0,40$ & Péssimo \\
\hline
\end{tabular}

\section{RESULTADOS E DISCUSSÃO}

Na Tabela 3 podem ser observados os valores de EPE, EPEa, $d, r$ e c para os municípios de Carira, Itabaianinha e Poço Verde do estado de Sergipe.

O método de Hargreaves-Samani foi o único a apresentar um bom desempenho de acordo com a classificação proposta por Camargo \& Sentelhas (Tabela 2) para as cidades de Carira e Itabaianinha, e desempenho mediano para a cidade de Poço Verde, já os métodos de Camargo, Blaney-Criddle e Linacre não apresentaram resultados satisfatórios em relação ao desempenho (Tabela 3).

Tabela 3. Relação dos coeficientes analisados para os municípios do Estado de Sergipe, utilizando dados das estações automáticas dos municípios de Carira, Itabaianinha e Poço Verde

\begin{tabular}{|c|c|c|c|c|c|}
\hline $\mathbf{P M} / \ldots$ & EPE & EPEa & d & $\mathbf{R}$ & c \\
\hline \multicolumn{6}{|c|}{ Carira - Estação automatic } \\
\hline Camargo & 1,45 & 1,40 & 0,64 & 0,73 & 0,47 \\
\hline $\begin{array}{l}\text { Hargreaves \& } \\
\text { Samani }\end{array}$ & 1,04 & 0,87 & 0,81 & 0,83 & 0,67 \\
\hline $\begin{array}{l}\text { Blaney- } \\
\text { Criddle }\end{array}$ & 1,76 & 1,77 & 0,63 & 0,93 & 0,59 \\
\hline Linacre & 1,80 & 1,81 & 0,65 & 0,82 & 0,53 \\
\hline \multicolumn{6}{|c|}{ Itabaianinha - Estação automatic } \\
\hline Camargo & 0,79 & 0,63 & 0,77 & 0,68 & 0,52 \\
\hline $\begin{array}{l}\text { Hargreaves \& } \\
\text { Samani }\end{array}$ & 0,63 & 0,40 & 0,89 & 0,82 & 0,73 \\
\hline $\begin{array}{l}\text { Blaney- } \\
\text { Criddle }\end{array}$ & 1,50 & 1,48 & 0,61 & 0,91 & 0,56 \\
\hline Linacre & 1,17 & 1,09 & 0,70 & 0,81 & 0,57 \\
\hline \multicolumn{6}{|c|}{ Poço Verde - Estação automatic } \\
\hline Camargo & 0,99 & 0,83 & 0,65 & 0,53 & 0,34 \\
\hline $\begin{array}{l}\text { Hargreaves \& } \\
\text { Samani }\end{array}$ & 0,81 & 0,44 & 0,88 & 0,69 & 0,61 \\
\hline $\begin{array}{l}\text { Blaney- } \\
\text { Criddle }\end{array}$ & 1,53 & 1,51 & 0,63 & 0,92 & 0,54 \\
\hline Linacre & 1,30 & 1,09 & 0,71 & 0,74 & 0,52 \\
\hline
\end{tabular}

Na Tabela 3 podem ser observados que os valores de EPE, com base em valores diários de ETo, apresentaram uma variação de 1,04 a 1,80 $\mathrm{mm} \mathrm{dia}^{-1}$, para o município de Carira, com os menores valores para o método de Hargreaves-Samani e os maiores valores para o método de Linacre. A variação média dos valores de ETo estimados pelos diferentes métodos e corrigidos pelos coeficientes de regressão linear, foi de 0,87 a 1,81 $\mathrm{mm} \mathrm{dia}^{-1}$, com os menores valores obtidos pelo método de Hargreaves-Samani e os maiores valores pelo método de Linacre. Os valores de EPEa para os métodos de Camargo e Hargreaves-Samani foram reduzidos, o que indica que a regressão linear melhorou o ajuste. 
Para os municípios de Itabaianinha e Poço Verde, os valores de EPEa para todos os métodos foram reduzidos, o que indica que a regressão linear melhorou o ajuste.

Nas Figuras 1, 2 e 3 podem ser observadas as relações entre os valores calculados de ETo pelos métodos de Hargreaves-Samani e Blaney-Criddle, com os valores obtidos pelo método padrão de Penman-Monteith, respectivamente, para os municípios de Carira, Itabaianinha e Poço Verde no período de maio de 2014 a abril de 2017.
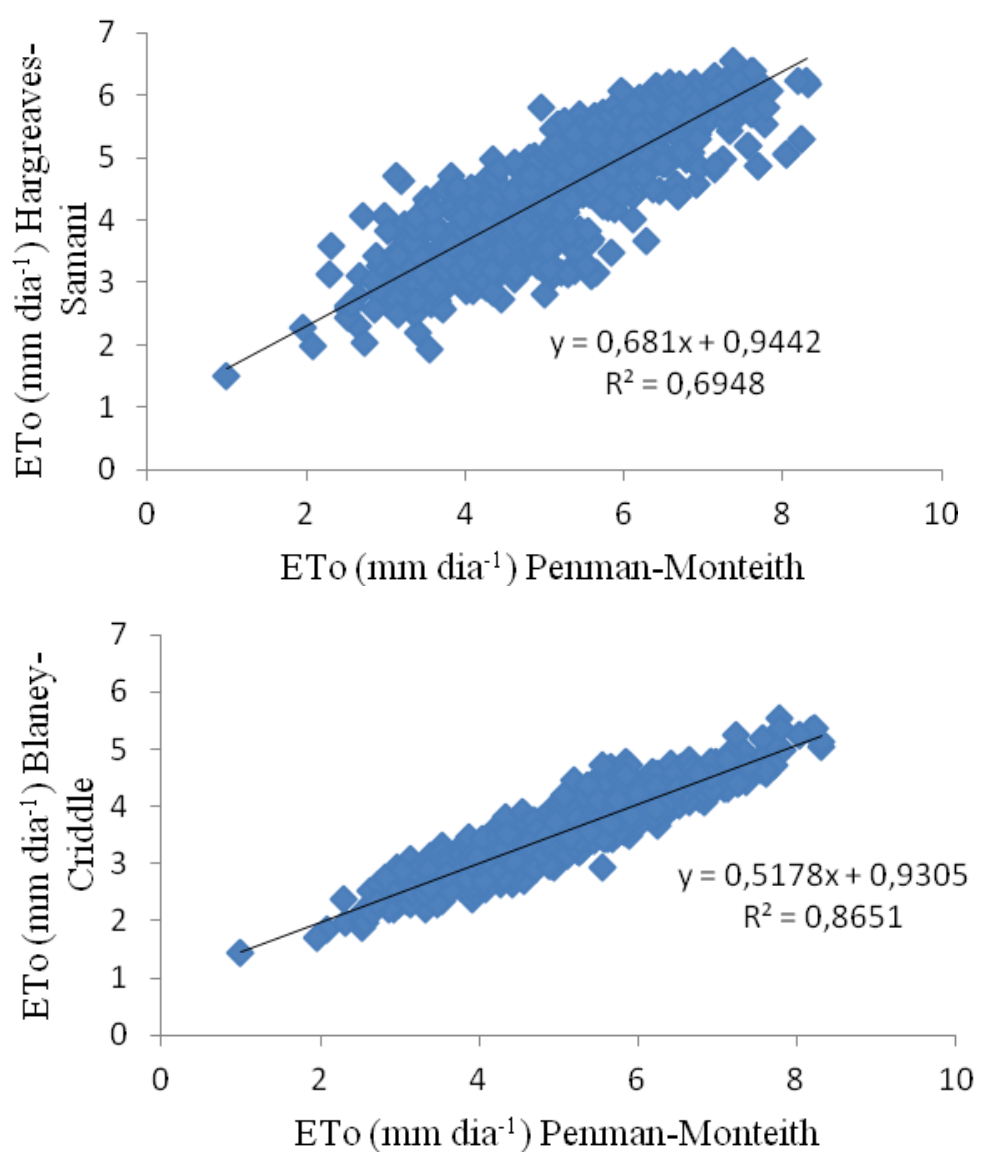

Figura 1 - Relações entre os valores mensais da evapotranspiração de referência calculados pelos métodos de Hargreaves \& Samani e Blaney-Criddle com os valores obtidos pelo método padrão de Penman-Monteith-FAO, para o município de Carira. 

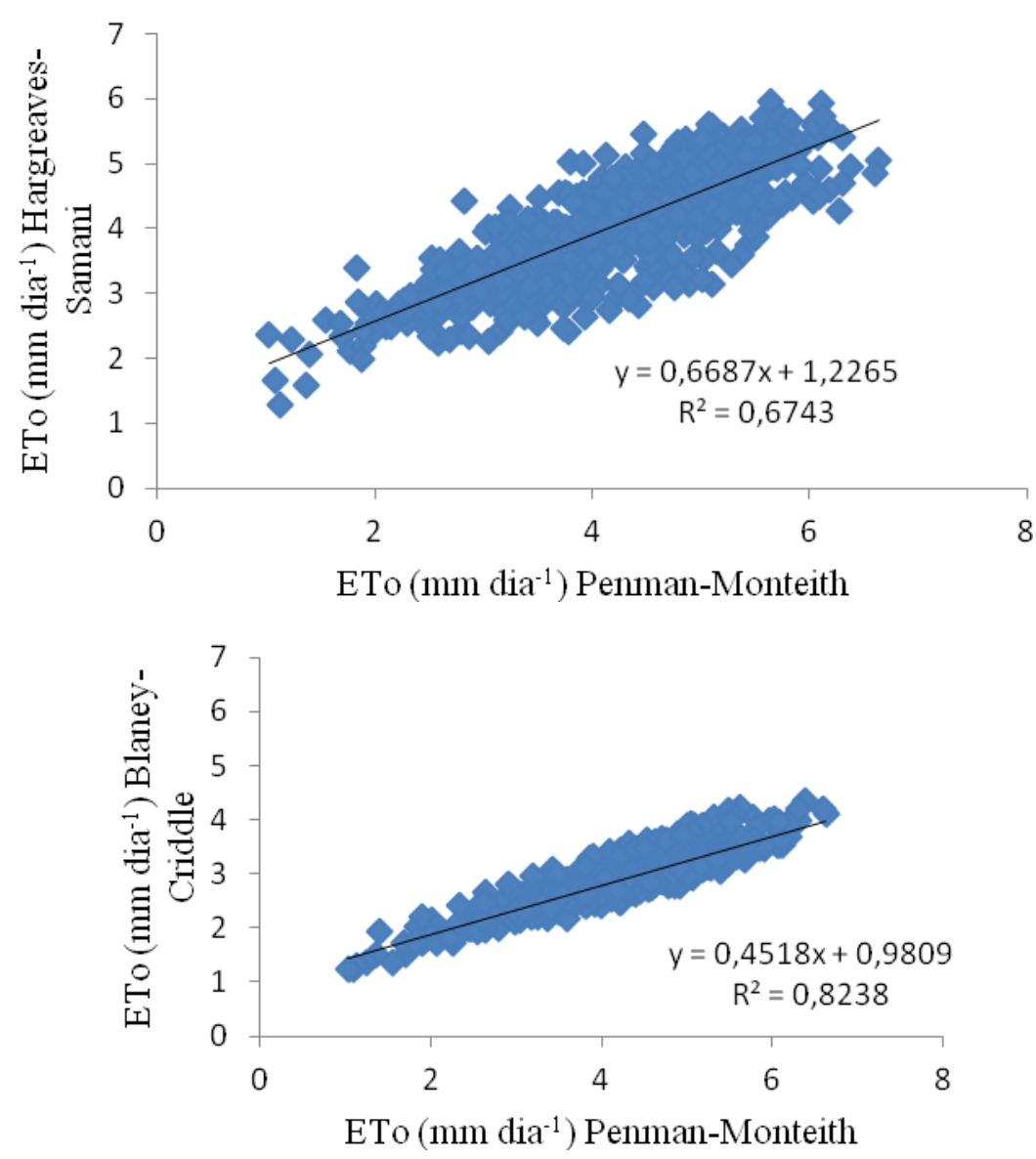

Figura 2 - Relações entre os valores mensais da evapotranspiração de referência calculados pelos métodos de Hargreaves \& Samani e Blaney-Criddle com os valores obtidos pelo método padrão de Penman-Monteith-FAO, para o município de Itabaianinha. 

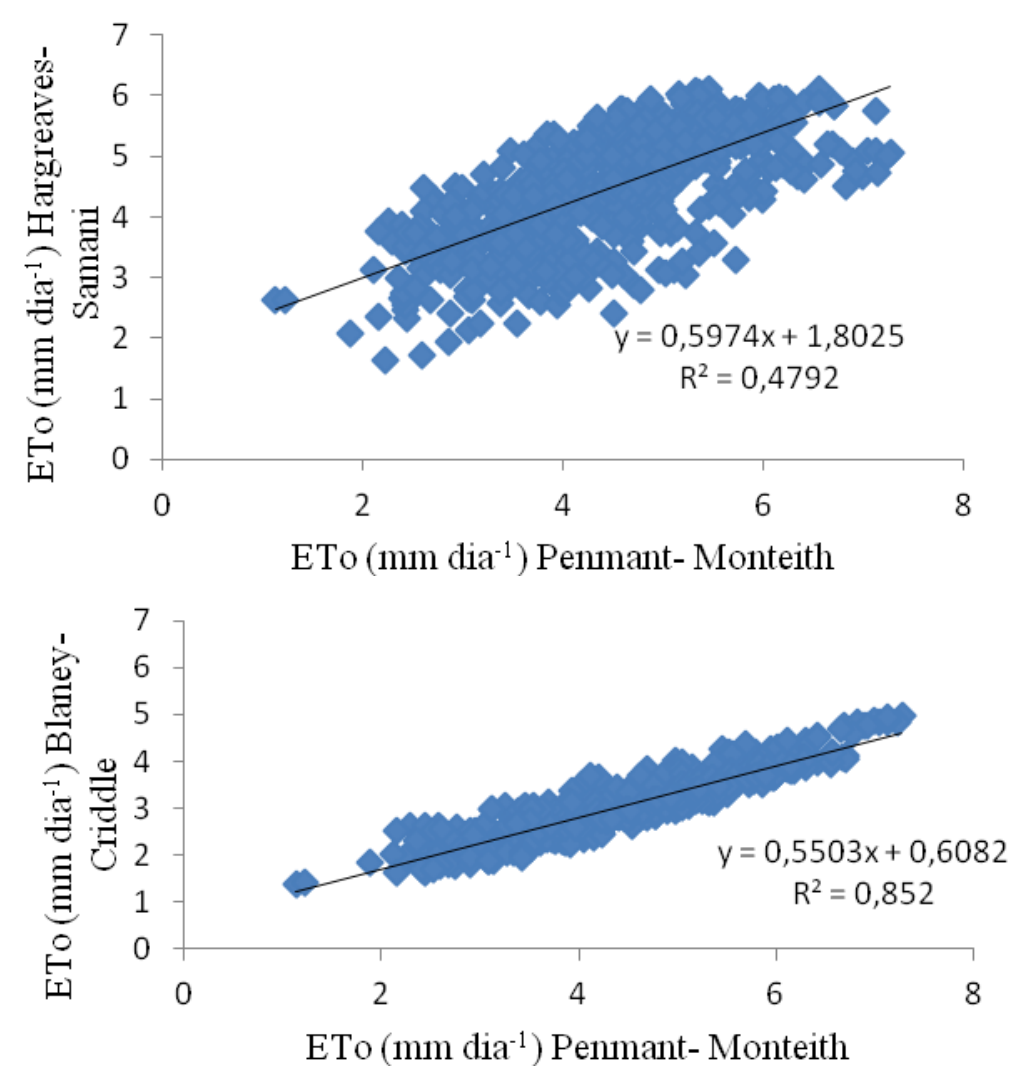

Figura 3 - Relações entre os valores mensais da evapotranspiração de referência calculados pelos métodos de Hargreaves \& Samani e Blaney-Criddle com os valores obtidos pelo método padrão de Penman-Monteith-FAO, para o município de Poço Verde.

Verificou-se uma correlação quase perfeita nos valores de ETo obtidos pelos métodos de Blaney-Criddle para os municípios de Carira, Itabaianinha e Poço Verde com os obtidos pelo método de Penman-Monteith, de acordo com a classificação da Tabela 1. Para o método de Hargreaves-Samani verificou-se uma correlação muito alta para os municípios de Carira e Itabaianinha, enquanto que para o município de Poço Verde a correlação foi classificada como alta.

Batista et al., 2007 [17] compararam os métodos de Penman 63, FAO-Penman corrigido, FAO radiação, Blaney-Criddle e Hargreaves-Samani com o método padrão Penman-Monteith para a região de Canindé do São Francisco - SE. Verificaram que o método de Blaney-Criddle apresentou melhores ajustes com o método de Penman-Monteith, obtendo resultados semelhantes aos obtidos para os municípios de Carira, Itabaianinha e Poço Verde.

\section{CONCLUSÃO}

Os métodos de Hargreaves-Samani e Blaney-Criddle tiveram altas correlações na estimativa da ETo com o método padrão FAO (Penman-Monteith). A utilização dos métodos de estimativa da ETo no manejo da irrigação pode proporcionar uma aplicação racional da água, reduzindo o desperdício na região.

O método de Hargreaves \& Samani utilizado na estimativa da ETo para os municípios de Carira, Itabaianinha e Poço Verde apresentou desempenho superior aos demais métodos estudados, podendo ser utilizado nas condições climáticas da região.

\section{AGRADECIMENTOS}

Ao programa CNPq/PIBIC pela concessão da bolsa de Iniciação Científica. 


\section{REFERÊNCIAS BIBLIOGRÁFICAS}

1. Cardoso MAG, Marcelino BC. Estimativa sazonal da evapotranspiração regional para o Rio Grande do Sul. In: SIMPÓSIO BRASILEIRO DE RECURSOS HÍDRICOS, XVI, João Pessoa, 2005 Nov 20-24; João Pessoa, PB.

2. Borges AC, Mendiondo EM. Comparação entre equações empíricas para estimativa da evapotranspiração de referência na Bacia do Rio Jacupiranga. Revista Brasileira de Engenharia Agrícola e Ambiental. 2007 Mai/Jun;11(3):293-300, doi:10.1590/S1415-43662007000300008.

3. Campos JHBC, Silva VPR, Azevedo PV, Borges CJR, Soares JM, Moura MSB, Silva BB. Evapotranspiração e produtividade da mangueira sob diferentes tratamentos de irrigação. Revista Brasileira de Engenharia Agrícola e Ambiental. 2008 Mar/Abr;12(2):150-156, doi:10.1590/S141543662008000200007.

4. Gonçalves FM, Feitosa HO, Carvalho CM, Gomes Filho RR, Valnir Junior M. Comparação de métodos da estimativa da evapotranspiração de referência para o município de Sobral-CE. Revista Brasileira de Agricultura Irrigada, 2009 Out;3(2):71-77, doi:10.7127/rbai.v3n200016

5. Suleiman AA, Hoogenboom G. Comparison of Priestley-Taylor and FAO-56 Penman-Monteith for daily reference evapotranspiration estimation in Georgia. Journal of Irrigation and Drainage Engineering, 2007;33(2):175-182, https://doi.org/10.1061/(ASCE)0733-9437(2007)133:2(175).

6. IBGE. Produção Agrícola Municipal. 2015. Disponível em: http://www.cidades.ibge.gov.br/. Acesso em 21 de abril de 2017.

7. Camargo AP. Balanço hídrico no Estado de São Paulo. 3.ed. Campinas: IAC; 1971. 24p. (Boletim Técnico, 116).

8. Thornthwaite $\mathrm{CW}$. An approach toward a rational classification of climate. Geographical Review, New York. 1948;38(1):55-94.

9. Allen RG, Pereira LS, Raes D, Smith M. Crop evapotranspiration: guidelines for computing crop water requirements. FAO: Rome; 1998. 300p. (Irrigation and Drainage Paper, 56).

10. Doorenbos J, Pruitt W. Guidelines for predicting crop water requirements. FAO: Rome; 1984. 178p. (Irrigation and Drainage Paper, 24).

11. Melo GL, Fernandes ALT. Evaluation of empirical methods to estimate reference evapotranspiration in Uberaba, State of Minas Gerais, Brazil. Engenharia Agrícola, Jaboticabal, 2012;32(5):875-888, http://dx.doi.org/10.1590/S0100-69162012000500007.

12. Linacre ET. A simple formula for estimating evapotranspiration rates in various climates, using temperature data alone. Agricultural Meteorology. 1977;18(1):409-424.

13. Fernandes ALT, Fraga Júnior EF, Takay BY. Avaliação do método Penman-Piche para a estimativa da evapotranspiração de referência em Uberaba - MG. Revista Brasileira de Engenharia Agrícola e Ambiental, Campina Grande. 2011 Mar;15(3):270-276,doi: 10.1590/S1415-43662011000300008.

14. Jensen ME, Burman RD, Allen RG. Evapotranspiration and Irrigation Water Requirements. ASCE Manuals and Reports on Engineering Practice No. 70, Am. Soc. Civil Engr., New York, NY; 1990. 332 pp, https://doi.org/10.1061/9780784414057

15. Willmott CJ. On the validation of models. Physical Geography. 1985;2:184-194, doi/abs/10.1080/02723646.1981.10642213

16. Camargo AP, Sentelhas PC. Avaliação do desempenho de diferentes métodos de estimativa da evapotranspiração potencial no Estado de São Paulo. Revista Brasileira de Agrometeorologia, 1997;5:89-97.

17. Batista WRM, Faccioli GG, Silva AAG. Determinação e comparação entre métodos de estimativas de evapotranspiração de referência para região de Canindé do São Francisco-SE. Revista da FAPESE. 2007;3(2):71-76. 